\title{
AIDS Clinical Trial
}

National Cancer Institute

\section{Source}

National Cancer Institute. AIDS Clinical Trial. NCI Thesaurus. Code C51359.

A clinical trial designed to evaluate the development, safety, and efficacy of vaccines and therapeutics directed against AIDS. 